\title{
Metal insulator transition in modulated quantum Hall systems
}

\author{
M. Koshino ${ }^{1}$ and T. Ando \\ Department of Physics, Tokyo Institute of Technology 2-12-1 Ookayama, Meguro-ku, Tokyo 152-8551, Japan
}

\begin{abstract}
The quantum Hall effect is studied numerically in modulated two-dimensional electron systems in the presence of disorder. Based on the scaling property of the Hall conductivity as well as the localization length, the critical energies where the states are extended are identified. We find that the critical energies, which are distributed to each of the subbands, combine into one when the disorder becomes strong, in the way depending on the symmetry of the disorder and/or the periodic potential.
\end{abstract}

Key words: quantum Hall effect, the Hofstadter butterfly, Anderson localization PACS: 73.43.-f, 71.30.+h

\section{Introduction}

The localization due to the disorder plays a crucial role in the integer quantum Hall effect. In a twodimensional (2D) electron system in strong magnetic fields, the weak disorder makes almost all the states localized, and the Hall current is carried only by the extended states left at the center of the Landau band. The Hall conductivity is exactly quantized when the Fermi energy lies on the localized region.

When the 2D electron system is subjected to a two-dimensionally modulated potential, the energy spectrum has a recursive gap structure called the Hofstadter butterfly in each of the Landau levels [1]. The system exhibits the quantum Hall effect when the Fermi energy is in each of those gaps, where the intricate gap structure leads to a nontrivial sequence of the quantized Hall conductivity [2]. The nonmonotonic behavior of the Hall conductivity peculiar to this system

\footnotetext{
1 Corresponding author.

E-mail: koshino@stat.phys.titech.ac.jp
}

was experimentally observed in lateral superlattices patterned on GaAs/AlGaAs heterostructures [3].

It is an intriguing problem how the Hall conductivity is quantized when the disorder is introduced to the Hofstadter butterfly. The localization problems in the disordered butterfly system have been studied by several authors. A finite-size scaling analysis was performed and the critical exponent was estimated at the center of the Landau level [5]. A qualitative discussion on the evolution of the extended states in the Hofstadter butterfly as a function of the disorder for several flux states was given $[6,7]$.

Recently the Hall conductivity $\sigma_{x y}$ was calculated and the effect of the localization on the quantum Hall effect was studied in the Hofstadter butterfly [9]. It was shown that $\sigma_{x y}$ becomes independent of the system size at $\sigma_{x y}=1 / 2$ (in units of $-e^{2} / h$ ), and those fixed points can be identified as the critical energies in an infinite system. While the systems in the previous work have an electron-hole symmetry between positive and negative energies, we study here the case without this symmetry to see whether the fixed points are still 
on $\sigma_{x y}=1 / 2$, and how the asymmetry affects the evolution of the critical energies as a function of disorder. The electron-hole symmetry occurs when both the periodic and the disorder potentials are symmetric with respect to zero energy. We first consider a system with an asymmetric disorder potential containing only positive scatters, while the periodic potential is left symmetric. Second, we recover the symmetry for the disorder, but make the periodic potential asymmetric as expected in the presence of the electron screening.

\section{Formulation}

We consider a two-dimensional system in a strong magnetic field with a periodic potential $V_{p}$ and a disorder potential $V_{d}$,

$$
H=\frac{1}{2 m}(\boldsymbol{p}+e \boldsymbol{A})^{2}+V_{p}+V_{d}
$$

The band structure is characterized by a parameter $\phi=B a^{2} /(h / e)$, a number of magnetic flux quanta penetrating unit cell [1]. We assume that $V_{p}$ has a square form

$V_{p}=V\left(\cos \frac{2 \pi}{a} x+\cos \frac{2 \pi}{a} y\right)+V^{\prime}\left(\cos \frac{4 \pi}{a} x+\cos \frac{4 \pi}{a} y\right)$,

where $V^{\prime}$ represents a double period component breaking the electron-hole symmetry between positive and negative energies. The disorder potential is taken as randomly distributed delta-potentials $\pm v_{0}$, where the amounts of the positive and negative scatterers are given by $N_{+}$and $N_{-}$, respectively. The energy scale for the disorder is given by $\Gamma=4 n_{i} v_{0}^{2} /\left(2 \pi l^{2}\right)$, where $l$ is the magnetic length and $n_{i}$ is the number of the scatterers in a unit area. We consider only the lowest Landau level, assuming that the magnetic field is strong enough and the mixing of the Landau levels is neglected.

We calculate the Hall conductivity using the Kubo formula for zero temperature,

$\sigma_{x y}=\frac{\hbar e^{2}}{i L^{2}} \sum_{\epsilon_{\alpha}<E_{F}} \sum_{\beta \neq \alpha} \frac{\left\langle\alpha\left|v_{x}\right| \beta\right\rangle\left\langle\beta\left|v_{y}\right| \alpha\right\rangle-\left\langle\alpha\left|v_{y}\right| \beta\right\rangle\left\langle\beta\left|v_{x}\right| \alpha\right\rangle}{\left(\epsilon_{\alpha}-\epsilon_{\beta}\right)^{2}}$,

where $\epsilon_{\alpha}$ is the energy of the eigenstate $|\alpha\rangle, E_{F}$ the Fermi energy, $v_{i}$ the velocity operator, and $L$ is the system size.

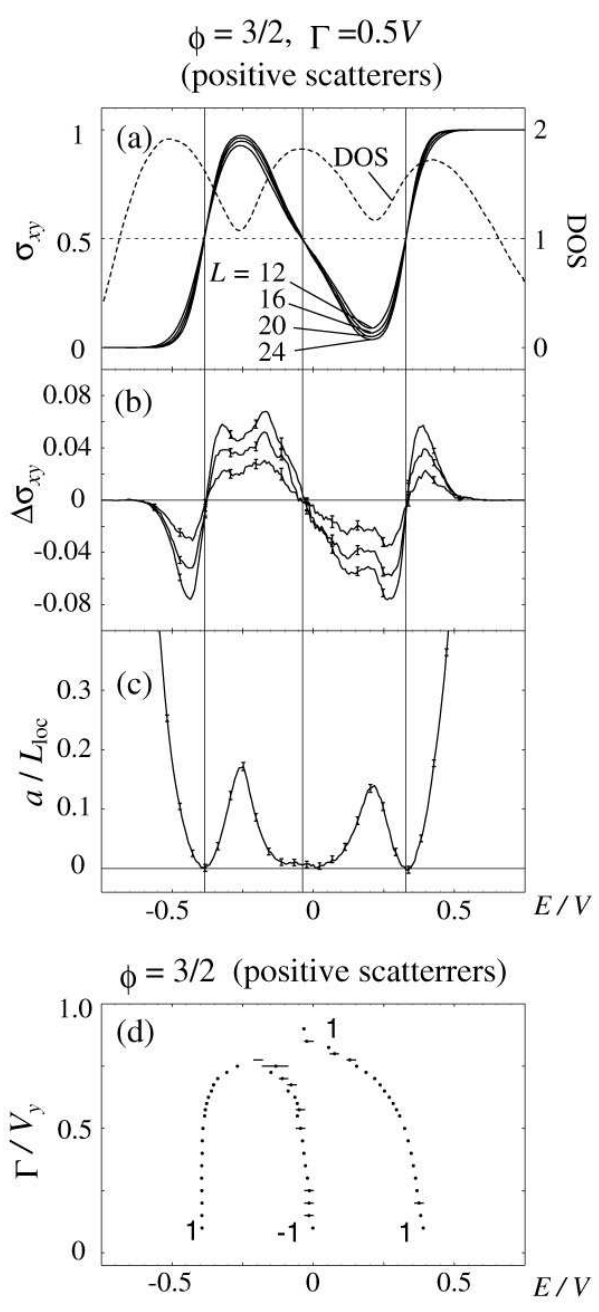

Fig. 1. (a) Hall conductivity $\sigma_{x y}$ (in units of $-e^{2} / h$ ) calculated for disordered systems containing positive scatterers only, with $\Gamma / V=0.5, \phi=3 / 2$ and $L / a=12,16,20$, 24. Dashed line represents the density of states in units of $1 /\left(V a^{2}\right)$. (b) Relative values of $\sigma_{x y}$ measured from the smallest $(L / a=12)$ sample. (c) Inverse localization length estimated in a Thouless number method. Vertical lines penetrating the panels represents the energies of $\sigma_{x y}=1 / 2$. (d) Trajectories of the critical energies as a function of $\Gamma$. Numbers represent the corresponding Hall conductivities. Horizontal bars show the energy regions where the error bar of the Hall conductivity reaches $\sigma_{x y}=1 / 2$.

\section{Results}

We first consider a system with symmetric potential $V^{\prime}=0$ and disorder potential containing only positive scatterers, where the latter breaks the electron-hole 


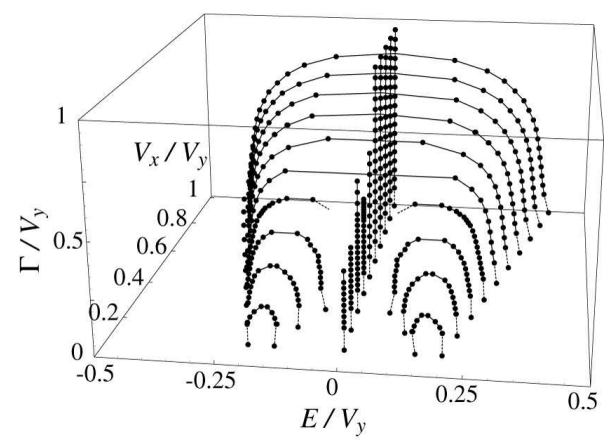

Fig. 2. Trajectories of the energies of $\sigma_{x y}=1 / 2$ as a function of $\Gamma$ and the anisotropy $V_{x} / V_{y}$, for a flux $\phi=3 / 2$ [9]. The points at $\Gamma=0$ show the energies of $\sigma_{x y}=1 / 2$ in clean systems. At $V_{x} / V_{y}=0.4$, we omit the points $0<E / V_{y}<0.2$ because it is numerically difficult to resolve the points around $E=0$.

(a)

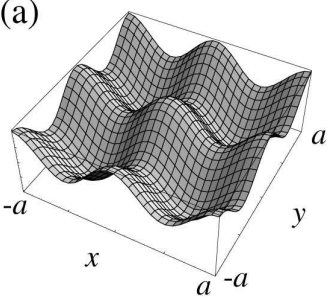

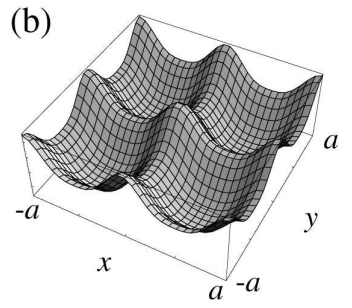

Fig. 3. Plot of the periodic potential $V_{p}(x, y)$ with (a) $V^{\prime} / V=0$ and (b) 0.2 .

symmetry. We assume a magnetic flux $\phi=3 / 2$, where the lowest Landau level splits into three subbands in the absence of disorder and $\sigma_{x y}$ for each of them becomes $(1,-1,1)$ (in units of $-e^{2} / h$ ) [2].

Figure 1 shows numerical results calculated for the disordered systems with $\Gamma=0.5 \mathrm{~V}$. We show in the panel (a) $\sigma_{x y}$ for several system sizes with the density of states, (b) the difference in $\sigma_{x y}$ measured from the smallest sample, and (c) the inverse localization length $1 / L_{\text {loc }}$ estimated by the Thouless number method [8], where every quantity is averaged over a number of different samples. We can immediately see that each subband has fixed points in the Hall conductivity at $\sigma_{x y}=$ $1 / 2$, and they agree with the energies where localization length diverges within the statistics error. $\sigma_{x y}$ off the fixed points always approaches 1 in the area $\sigma_{x y}>$ $1 / 2$, and 0 in $\sigma_{x y}<1 / 2$, leading to the Hall plateau in an infinite system. Therefore it is natural to identify the positions of the extended states as the points of $\sigma_{x y}=1 / 2$.

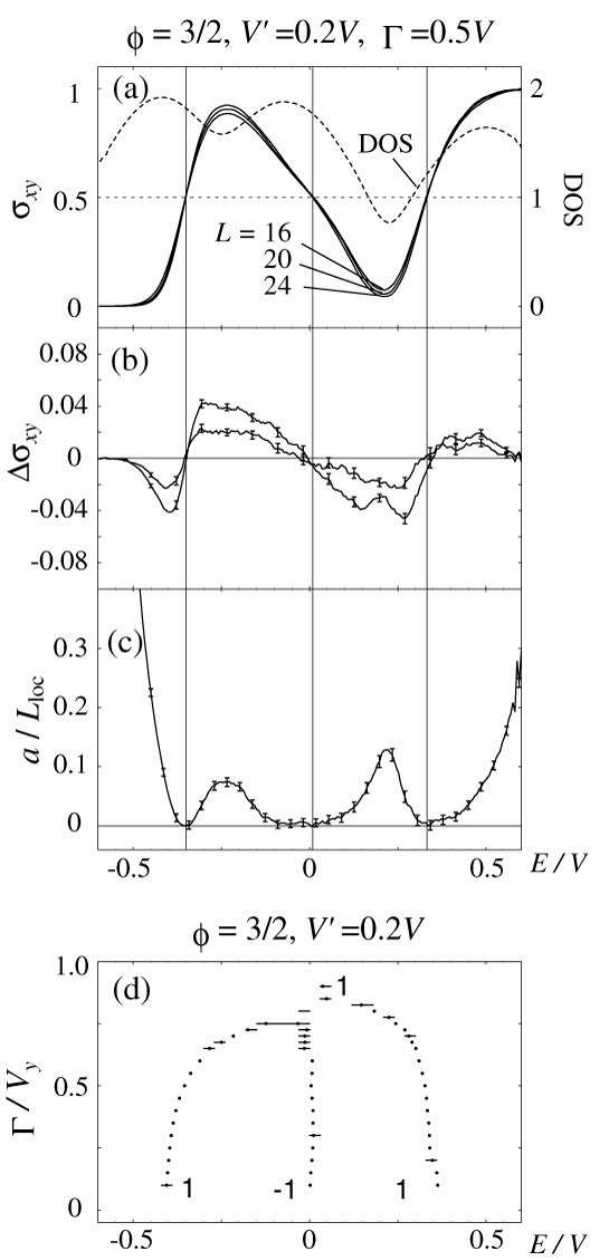

Fig. 4. Plots similar to Fig. 1 calculated for a system with an asymmetric modulation $V^{\prime}=0.2 \mathrm{~V}, \Gamma / V=0.5, \phi=3 / 2$ and $L / a=16,20,24$.

When we increase the strength of the disorder, the subband structure becomes obscure and the Hall plateau inside the subband gaps should disappear. The panel (d) in Fig. 1 shows the trace of the critical energies as a function of $\Gamma$, which are identified as the positions of $\sigma_{x y}=1 / 2$. We can see that three energies with the Hall integer $1,-1,1$ become closer as the disorder increased, and the lowest and the center are united at $\Gamma \approx 0.8 \mathrm{~V}$ and the upper is left with the Hall conductivity +1 .

If we distribute equal amounts of positive and negative scatterers, three critical energies combine all together at $E=0$ due to the electron-hole symmetry. In Fig. 2, we show the trace of the critical energies calcu- 
lated for the symmetric cases for comparison [9]. We also show the results for anisotropic modulations $V_{p}=$ $V_{x} \cos (2 \pi x / a)+V_{y} \cos (2 \pi y / a)$ with $V_{x} \neq V_{y}$. When we change the modulation from $2 \mathrm{D}\left(V_{x} / V_{y}=1\right)$ toward 1D $\left(V_{x} / V_{y}=0\right)$ with fixed $\Gamma$, the critical energy for the center subband branches into three at $V_{x} / V_{y} \approx 0.4$. Then we have five critical energies for $V_{x} / V_{y}<0.4$, even though there are only three subbands [9].

The electron-hole symmetry breaks as well when the modulation potential itself lacks the symmetry with respect to zero energy. We consider here a system which has a double period component $V^{\prime}=0.2 \mathrm{~V}$ in the periodic potential. The amounts of positive and negative scatterers are assumed to be equal. The periodic potential is shown in Fig. 3, where the potential becomes flat at bottom and sharp on top. The potential in a real system actually has this feature due to the screening by the electrons, even when the external modulation is symmetric [10].

The numerical results are presented in Fig. 4. The Hall conductivity has fixed points at $\sigma_{x y}=1 / 2$ in the middle and the lower subband, while it is hard to decide one for the upper subband due to a large statistic error. Possibly this difficulty comes from the low density of states around this region. The trajectories of the critical energies, the panel (d), shows that the center branch is slightly closer to the upper than to the lower in the region $\Gamma \lesssim 0.5$, even though the upper subband is a little apart from the other two as seen in the density of states (a). When $\Gamma$ becomes larger, however, it is likely that the center branch combines with the lower, similarly to the previous case. In both cases we have considered, the pair annihilation occurs to the pair on the side of higher density of states.

\section{Summary and Conclusion}

To summarize, we calculated the Hall conductivity and the localization length in the systems without the electron-hole symmetry. We considered two different cases, where we introduce the asymmetry to the disorder potential or to the periodic modulation. In both cases, we found fixed points in the Hall conductivity at $\sigma_{x y}=1 / 2$, and that those points coincide with the divergence of the localization length within the statistics error. The evolution of the critical energies also becomes asymmetric in each case in such a way that two branches on the side with a higher density of states combine together and one remains intact. We note that, to detect the asymmetry proposed here, the magnetic field should be strong enough that Landau levels are well isolated, since the asymmetry is also caused by the coupling among different Landau levels. We expect that detecting the asymmetry in $\sigma_{x y}$ provides a possible probe for the electron screening in modulated quantum Hall systems.

Acknowledgments This work has been supported in part by a 21st Century COE Program at Tokyo Tech "Nanometer-Scale Quantum Physics" and by Grantsin-Aid for Scientific Research and for COE (12CE2004 "Control of Electrons by Quantum Dot Structures and Its Application to Advanced Electronics"), and Scientific Reseach from the Ministry of Education, Science and Culture, Japan. Numerical calculations were performed in part using the facilities of the Supercomputer Center, Institute for Solid State Physics, University of Tokyo.

\section{References}

[1] D. R. Hofstadter, Phys. Rev. B 14 (1976) 2239.

[2] D. J. Thouless: M. Kohmoto, M. P. Nightingale, and M. den Nijs, Phys. Rev. Lett. 49 (1982) 405.

[3] C. Albrecht, J. H. Smet, K. von Klitzing, D. Weiss, V. Umansky, and H. Schweizer, Phys. Rev. Lett. 86 (2001) 147.

[4] M. C. Geisler, J. H. Smet, V. Umansky, K. von Klitzing, B. Naundorf, R. Ketzmerick and H. Schweizer, Phys. Rev. Lett. 92 (2004) 256801.

[5] B. Huckestein, Phys. Rev. Lett. 72 (1994) 1080.

[6] Y. Tan, J. Phys. Condens. Matter 6 (1994) 7941; Phys. Rev. B 49 (1994) 1827.

[7] K. Yang and R. N. Bhatt, Phys. Rev. B 59 (1999) 8144.

[8] T. Ando, J. Phys. Soc. Jpn. 52 (1983) 1740.

[9] M. Koshino and T. Ando, J. Phys. Soc. Jpn. 73 (2004) 3243. 
[10] See, for example, T. Suzuki and T. Ando, J. Phys. Soc. Jpn. 62 (1993) 2986 and references cited therein. 